\title{
CFD analysis of a full-scale ceramic kiln module under actual operating conditions
}

\author{
Massimo Milani ${ }^{1}$, Luca Montorsi $^{1, *}$, Matteo Stefani ${ }^{1}$, and Matteo Venturelli ${ }^{1}$ \\ ${ }^{1}$ University of Modena and Reggio Emilia - Department of Sciences and Methods for Engineering - \\ via Amendola 2 - Pad. -Morselli, 42122 Reggio Emilia, Italy
}

\begin{abstract}
The paper focuses on the CFD analysis of a full-scale module of an industrial ceramic kiln under actual operating conditions. The multi-dimensional analysis includes the real geometry of a ceramic kiln module employed in the preheating and firing sections and investigates the heat transfer between the tiles and the burners' flame as well as the many components that comprise the module. Particular attention is devoted to the simulation of the convective flow field in the upper and lower chambers and to the effects of radiation on the different materials is addressed. The assessment of the radiation contribution to the tiles temperature is paramount to the improvement of the performance of the kiln in terms of energy efficiency and fuel consumption. The CFD analysis is combined to a lumped and distributed parameter model of the entire kiln in order to simulate the module behaviour at the boundaries under actual operating conditions. Finally, the CFD simulation is employed to address the effects of the module operating conditions on the tiles' temperature distribution in order to improve the temperature uniformity as well as to enhance the energy efficiency of the system and thus to reduce the fuel consumption.
\end{abstract}

\section{Introduction}

The ceramic industry is well known to be characterized by energy intense processes and among them the kiln is one of the main responsible of the primary energy consumption. Recent regulations for the energy consumption limit the fuel or electricity use [1] and therefore, the design of the kiln plays a fundamental role in meeting the regulations' requirements.

Due to the complex physical phenomena that take place in industrial ceramic kiln, simulation tools are an important instrument in the design process of the kiln; thus, different approaches have been adopted for the optimization of the kilns' performance. In [2] a theoretical formulation for the prediction of the kiln operating characteristics has been proposed under regime conditions and many simplifications to the physical phenomena had to be made in order to close the mathematical model. Similar approach is adopted also in [3] in which the main focus was the assessment of the heat transfer between the hot air flow and the kiln walls. The numerical simulation becomes a valuable tool in investigating the performance of complex physical systems including different physical phenomena. Lumped

*Corresponding author: luca.montorsi@unimore.it 
and distributed numerical analysis has been extensively adopted for the simulation of complex systems under time dependent operating conditions. In [4] a novel concept for the hydrogen production has been investigated by modelling the entire proposed plant and different operations have been compared in order to define the best energy efficiency configuration. Similarly, in [5] the numerical analysis of the entire cogeneration systems based on aluminium-water combustion was used to determine the best turbine operating point under different working conditions. The modelling approach has also been employed for the validation of integrated waste-to-energy systems with low environmental impact and the advantages compared to traditional technologies have been outlined [6]. Despite the dimensional approximation, the $0 \mathrm{D} / 1 \mathrm{D}$ models proved also to be able to account for complex physics involving heat transfer phenomena while including the layout of the full system. Nevertheless, the lumped and distributed parameter model has to assume a large number of quantities that can be difficult to derive from experimental measurements or theoretical approaches. Thus, the combination of this modelling approach to a full multi-dimensional approach of the involved physics is paramount in addressing accurately the behaviour of the analysed systems [7-9].

In this paper, the CFD simulation of the full geometry of a ceramic kiln model is carried out. A module of the preheating section as well as a module in the firing section are analysed. Insulating materials and alumina rollers and burners are included in the simulation and the effect on the tile temperature distribution is investigated. Furthermore, radiation is accounted for in the modelling in order to assess the contribution of the radiative heat transfer in the total heat absorbed by the ceramic tiles during the preheating and firing regions of the kiln. The results are useful not only to better model the heat transfer processes in the lumped and distributed parameter model of the real kiln, but also to better understand the main heat transfer contributions that influence the tiles' temperature profile and thus the product quality.

\section{Test case}

The ceramic kiln simulated in this paper is real production facility located in the ceramic district in Emilia Romagna - Italy. It is characterized by a production rate of approximately $5,000 \mathrm{~kg} / \mathrm{h}$ of tiles and it is designed for a continuous working load of about 8,700 hours per year. The entire ceramic kiln includes 43 modules with a length of $2.1 \mathrm{~m}$ each and it can be subdivided into the following 5 sections: pre-heating, firing, fast cooling, slow-cooling and outlet section, see Figure 1.

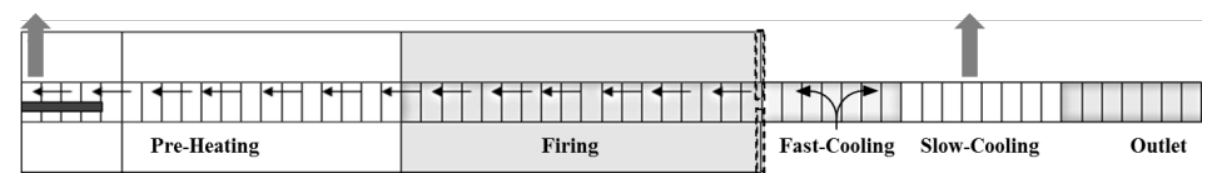

Fig. 1. Schematic of the entire ceramic kiln.

This study focuses on one module in the preheating section and one module in the firing section. These section are critical in order to reach the quality of the tiles and determine the energy consumption of the kiln. In particular, an accurate temperature control is mandatory for achieving the desired product quality and avoiding scraps.

In the pre-heating section, no burners are employed and the heating is due to the hot air flow from the burners' zone. As far as the energy consumption is concerned, the most important zone is the firing where the burners are installed. The burners are fuelled by natural gas and the fuel rate is controlled for each group of 8 burners. One group of burners belongs to two modules and there are two groups of burners for each module that are 
installed above and below the rollers' plane respectively. The different control of the burners in the chambers above and below the rollers is critical for achieving the correct temperatures in these volumes. At the end of the firing section, a fireproof wall is positioned in order to separate the cooling zone to the firing zone; main task of this wall is to partition the flow between the firing and fast-cooling sections. Its height is adjusted in order to always direct the flow from the cooling to the firing section and regulate the temperature at the interface of these two regions.

An important characteristic of the analysed kiln is the opposite direction of the hot air flow and the tile motion. A mentioned before, the air is inducted from the cooling zone by means of a fan located at the beginning of the kiln before the flue gas stack. Therefore, the pressure in the cooling zone is higher than the one in the firing section, which is usually characterized by values below the atmospheric pressure. Thus, the leakages in the walls of the modules allow the cold ambient air to enter the kiln chambers influencing the temperature of the hot air flow and thus the energy consumption of the entire system. This effect is not taken in the present analysis into account. The remaining portion of air in the fast-cooling section exits the kiln through the slow-cooling section and the final outlet stack. An important feature of the analysed kiln is the counter-current heat transfer mode.

\section{Numerical modelling}

The CFD analysis is carried out by means of the Star CCM+ code licensed by CD-Adapco. Particular care in the mesh construction as well as in the modeling of the heat transfer is paid. Ideal gas law is assumed to model the air thermal behavior, while the energy equation is solved for the enthalpy and coupled to the Navier-Stokes equation to solve the fluid flow. Dynamic simulations are performed in order to account also for the tiles' motion. Figure 2 shows the real geometry of the analysed modules, while Figures 3a) and 3b) depict the computational domains adopted for the simulation of the preheating module and the firing module respectively. The latter domain includes also the grid for the EKO burners, which are designed to preheat the oxidizing air by recovery the heat from the hot air flow drawn from the kiln chambers.

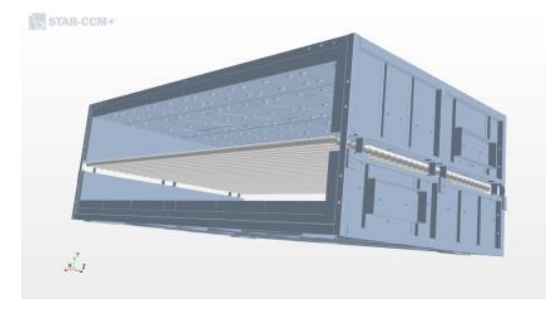

Fig. 2. Geometry of the analysed kiln's module.
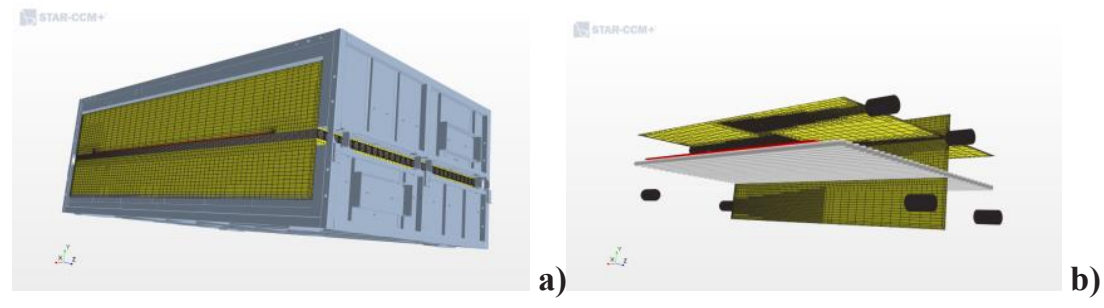

Fig. 3. Computational domain for the module in the a) preheating and b) firing sections. 
Both grids are constructed using a trimmed hexahedral mesh with proper refinements particularly in the regions where the burners' flame is supposed to take place, see Figure $3 b)$. The total number of cells used in the CFD domain is approximately 4 million. The mesh used in the simulation is the best trade-off between result's accuracy and computational effort. The fluid domains consist of the upper and lower chambers, the alumina rollers, the tiles' material and eventually the EKO burners. Turbulence is accounted for by means of the two zonal version of the $k-\omega$ model, known as the shear stress transport model [9]. This approach demonstrated in previous studies a good accuracy in predicting the flow field for multi-phase flows in hydraulic components [10].

The radiation contribution on the total heat transfer is calculated by using the Surface-to-Surface (S2S) radiation model that model the thermal radiative heat transfer between surfaces of arbitrary complexity that form enclosed spaces. The medium that fills the space between the surfaces is non-participating, thus, it does not absorb, emit, or scatter radiation. The radiation properties and the thermal boundary conditions that are imposed on each surface define uniquely the amount of radiation that a surface receives and emits. The radiation energy transfer to or from each surface is calculated from the radiation transport equation and the boundary conditions $[12,13]$.

The combustion process for the burners in the firing section module is modelled according to the Eddy Brake Up (EBU) approach. This model characterizes the reacting flow system by a specified number of species and chemical reactions. The individual species in the global reaction are assumed to be transported at different rates according to their own governing equations. The Standard EBU model uses Eqn. (1) as the governing transport equation. The reaction rate is modelled through an expression that takes the turbulent micromixing process into account by means of dimensional arguments. Thus, for a reaction of the form:

$$
v_{F} F+v_{O} O \rightarrow v_{P 1} P_{1}+v_{P 2} P_{2}+\ldots \ldots+v_{P j} P_{j}
$$

the rate of fuel depletion is assumed to be:

$$
\begin{gathered}
R_{F}=-\frac{\rho}{M_{F}}\left(\frac{1}{\tau_{R}}\right) A_{e b u} \min \left[\bar{Y}_{F}, \frac{\bar{Y}_{O}}{s_{O}}\right] \operatorname{moles} /\left(\mathrm{m}^{3} \mathrm{~s}\right) \\
s_{O} \equiv v_{O} M_{O} / v_{F} M_{F} ; s_{P i} \equiv\left|v_{P i}\right| M_{P i} / v_{F} M_{F} ; \tau_{R}=\tau_{m i x}=\frac{k}{\varepsilon}
\end{gathered}
$$

where $k$ is the turbulent kinetic energy and $\varepsilon$ its dissipation rate. The min operator on the right-hand side of Eqn. (2) indicates that the concentration of the limiting reactant should be used to determine a mass fraction scale when calculating the reactant consumption rate. Eqn. (2) essentially states that the integrated micromixing rate is proportional to the mean (macroscopic) concentration of the limiting reactant divided by the time scale of the large eddies.

Figure 4 shows the boundary conditions adopted for the simulations. Since many of the data necessary for properly setting the CFD boundary conditions were not available from experimental measurements, the values are derived from a previous simulation of the overall ceramic kiln under actual operations using a lumped and distributed parameter model described in [13]. In particular, the inlet mass flow rate for the upper and lower chambers as well as the relating outlet pressure values are taken in the $0 \mathrm{D} / 1 \mathrm{D}$ at the exact location as in the real module. 


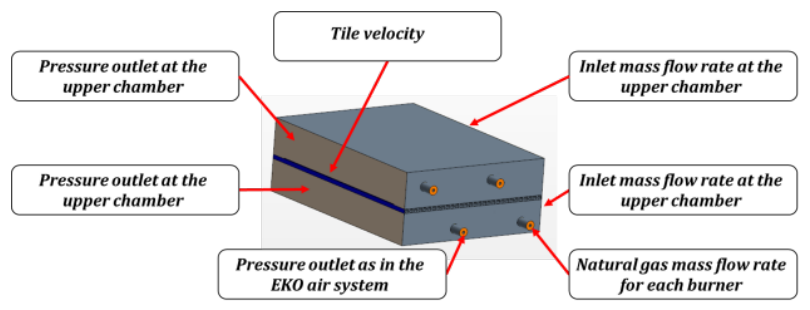

Fig. 4. Boundary conditions for the computational domain.

Furthermore, the lumped approach was used to estimate the natural gas flow rate for each burner for the module in the firing section of the kiln; this value would be very difficult to measure experimentally. The effect of the tile motion is also accounted for in the analysis using the sliding interface technique available in STAR-CCM + . Therefore, the thermal exchange between the tiles and the surrounding environment takes place through the connecting interfaces that updates on a time step basis to address the tile translation on the alumina rollers. The tile motion speed is set as in the real kiln, i.e. $0.35 \mathrm{~m} / \mathrm{s}$; therefore, it takes approximately $60 \mathrm{~s}$ for a single tile to go through the considered modules. The effects of the insulating material on the global thermal balance of the kiln module are determined by means of a global heat transfer coefficient at the walls. This heat transfer coefficient is calculated by including the convection with the ambient air on the external side of the kiln wall, $\alpha_{a m b}$, and the thermal conductivity through the different layers of insulation, $\lambda_{i}$, along with the corresponding layers' thickness, $t h_{i}$. The global thermal conductivity of the walls, $C_{T h \text {,wall, }}$, results as follows:

$$
C_{\text {Th,wall }}=\frac{1}{\frac{1}{\alpha_{a m b}}+\sum_{i}^{n_{\text {layers }}} \frac{t h_{i}}{\lambda_{i}}}
$$

\section{Results and discussion}

The detailed calculation of the flow field within the kiln module makes it possible to evaluate accurately the temperature distribution of the tiles as well as the air in the upper and lower chambers. In particular, the temperature distribution of the ceramic material should be as uniform as possible in order to enhance the quality of the final product. Figure 5 shows the temperature of the tiles on the top surface after $60 \mathrm{~s}$ of simulation time for both analysed modules. Figure 6 depicts the same results on the bottom surface. The tile top and bottom temperature distributions result to be not uniform and they are strongly affected by the hot air flow direction. If this effect could be expected for the preheating module, it is less trivial for the firing section. Furthermore, the lateral surface of the tiles is characterized by the highest temperature values, see Figure 7 . This result can be explained by the larger local surface to volume ratio of the tile side and by the effects of the extremes of the alumina rollers. In fact, the alumina rollers are at a higher temperature than the tile's one and close to the value of the hot air flow. Figure 7 shows also the interaction between the tiles and the rollers, since the alumina that is covered by the tiles is characterized by a lower temperature since the tile protect them from the radiation of the upper chamber and absorb heat due conduction through the contacting areas between them.

Figure 8 plots the temperature distribution on a cut plane through the axes of the burners that equips the module in the firing section of the kiln. The flames of the burners result strongly influenced by the hot air flow even though their velocity is relatively small compared to the flame one. 


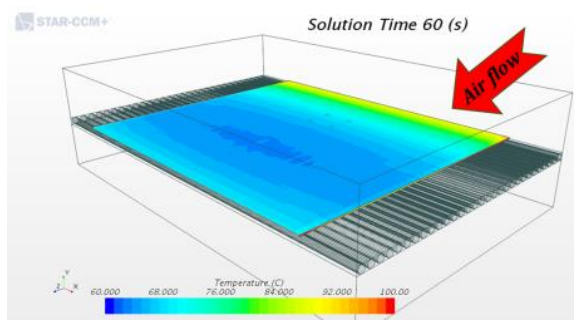

a)

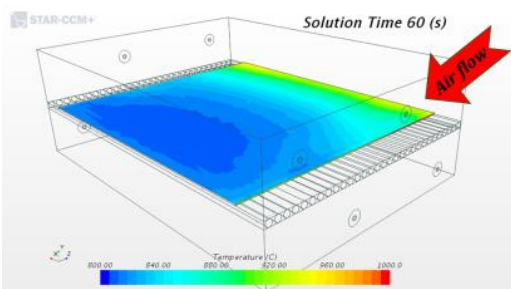

b)

Fig. 5. Temperature distribution on the top surface of the tile for the a) preheating module and b) the eight-burner module.

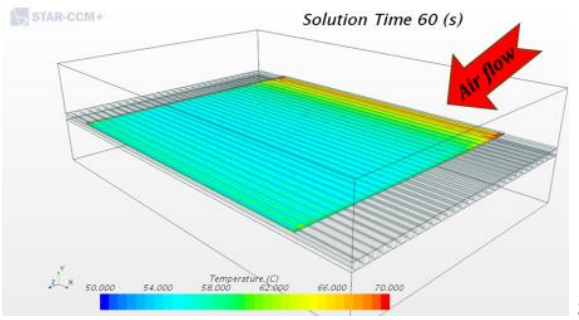

a)

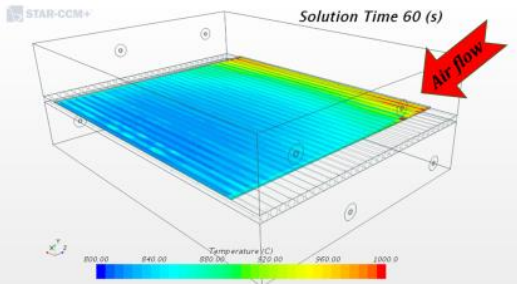

b)

Fig. 6. Temperature distribution on the bottom surface of the tile for the a) preheating module and b) the eight-burner module.
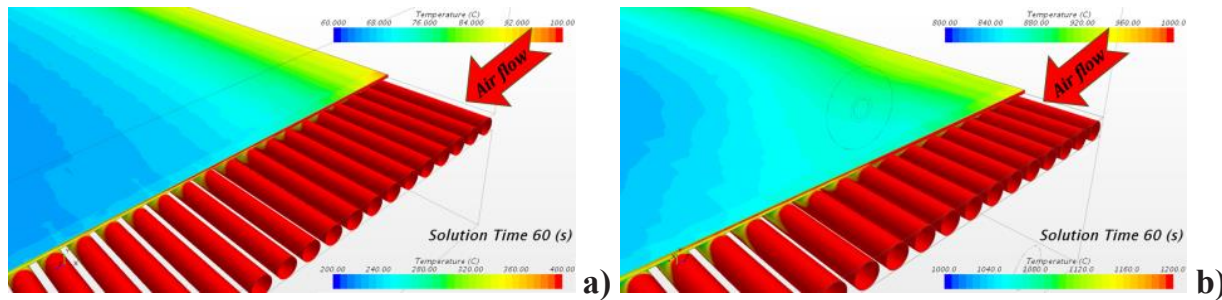

Fig. 7. Temperature distribution on the lateral surface of the tile and on the alumina rollers for the a) preheating module and $b$ ) the eight-burner module.
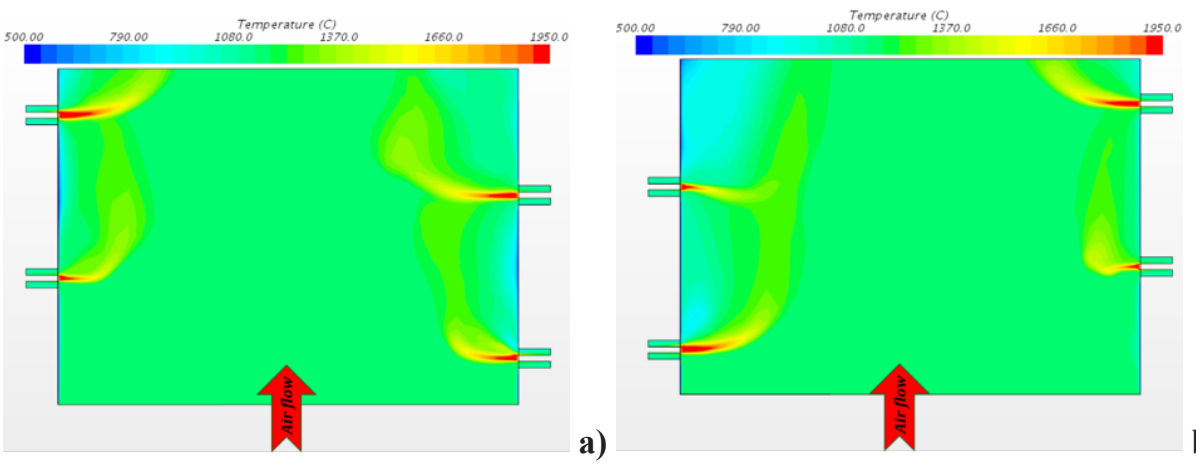

b)

Fig. 8. Temperature distribution on a cut plane through the burners' axis of a) the upper chamber and b) the lower chamber for the eight-burner module. 
Nevertheless, the central regions of the upper and lower chambers demonstrate to have a uniform temperature close to $1300^{\circ} \mathrm{C}$. Colder spots can be noticed close to the walls in between the burners' flames that act like a shield for the hot air stream. Therefore, recirculation regions form at low temperature due to the heat dissipation through the walls.

Figure 9 plots the time histories of the characteristic temperatures for the tile. The difference between the average value of the temperature on the top and bottom surfaces of the tile is close to $10^{\circ} \mathrm{C}$ for the preheating module and reaches $30^{\circ} \mathrm{C}$ in the firing module. This difference is very important to control, since it may influence significantly the quality of the product and it is very difficult to be measured experimentally. The simulations demonstrate that even though the thickness of the tile is small, i.e. less than $10 \mathrm{~mm}$, the temperature gradient between the top and the bottom of the tile is significant. Furthermore, the highest temperature in the tile surface is reached at the front side, where the tile is more strongly affected by the hot counter-current flow. The temperature at the side results to be approximately $40^{\circ} \mathrm{C}$ larger than the volume average temperature, whose value demonstrate to be close to the tile bottom temperature.

Finally, Figure 10 shows the time histories of the total heat transfer to the tile and the contribution of the radiative heat transfer. The heat radiated from the surrounding walls to the tiles results to be the main responsible of the total heat transfer, i.e. $81 \%$ and $72 \%$ for the preheating and firing modules respectively. In fact, the temperature difference between the tile surfaces and the surrounding ones remains very large in both analysed modules. Furthermore, the relative velocity of the flow and the tiles is rather slow radiative heat transfer, thus limiting the convection heat transfer from the hot stream to the tile surfaces. These figures add an insight in the thermal behaviour of the tile firing within the ceramic kiln; moreover, they are used to improve the accuracy of the predictive capabilities of the lumped and distributed parameter model. In fact, the view factors of the radiating surfaces are calculated in the $0 \mathrm{D} / 1 \mathrm{D}$ model in order to determine the correct contribution of radiation on the total heat transfer to the tile on the basis of this CFD result.
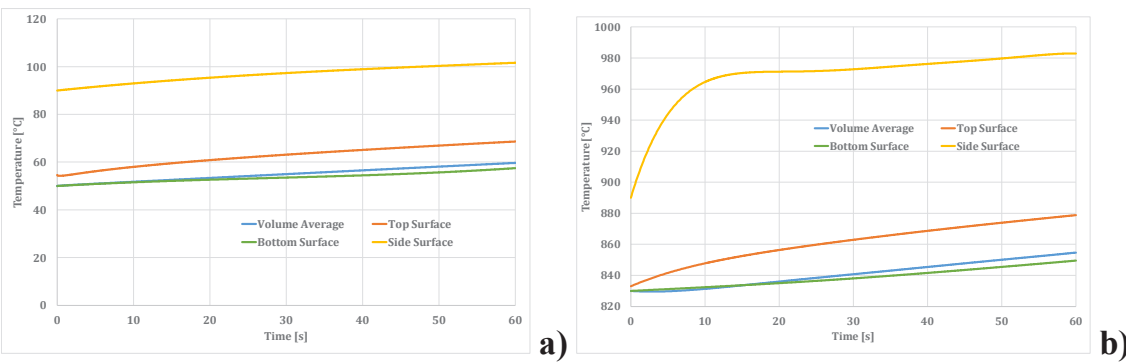

Fig. 9. Time histories of volume average temperature and of the tile surfaces' temperature for the a) preheating module and b) the eight-burner module.
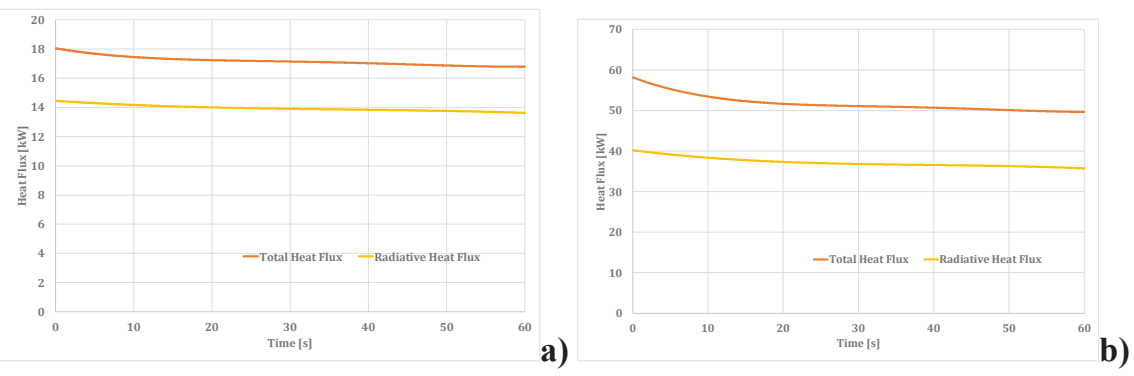

Fig. 10. Time histories of total heat flux absorbed by the tile and the contribution due to radiation the a) preheating module and b) the eight-burner module. 


\section{Conclusion}

In this paper, the CFD analysis of a full-scale module of an industrial ceramic kiln under actual operating conditions has been carried out. A module in the preheating section and one in the firing region have been investigated and the thermal behaviour of the tiles in these parts of the kiln has been assessed.

In the analysis the combustion in the burners of the firing module has been addressed by using the Eddy Brake Up model for the oxidation of natural gas with air; the Surface-to-Surface (S2S) radiation model has been adopted for calculating the thermal radiative heat transfer between surfaces of the ambient surrounding the tiles.

Numerical results demonstrated that the temperature distribution on the top and bottom surfaces of the tile is not uniform despite the small thickness and a difference of approximately $10^{\circ} \mathrm{C}$ and $30^{\circ} \mathrm{C}$ is highlighted in the preheating and in the firing modules respectively. The hottest part of the tile proved to be the front side surface with a temperature $40^{\circ} \mathrm{C}$ higher than the rest of the surfaces.

Finally, the contribution of the radiative heat transfer to the total heat absorbed by the tile was calculated to be close to $81 \%$ in the preheating module and to $72 \%$ in the firing section. This result is paramount for improving the predictive capabilities of the lumped and distributed parameter model of the entire kiln, as well as to address the thermal behaviour of the tile firing process.

The present work is part of Design for Resource and Energy efficiency in CerAMic Kilns (DREAM) project. The project has received funding from the European Union's Horizon 2020 research and innovation programme under grant agreement No 723641.

\section{References}

1. A. Christos, T, Theocharis, Appl. Therm. Eng. 21, 1231-1249 (2001)

2. S. Kaya, E. Mançuhan, K. Küçükada, Appl. Energ. 86, 325-332 (2009)

3. A. Mezquita, J. Boix, E. Monfort, G. Mallol, Appl. Therm. Eng. 65, 102-110 (2014)

4. S. Mercati, M. Milani, L. Montorsi, F. Paltrinieri, Appl. Energ. 97, 686-694 (2012)

5. F. Franzoni, S. Mercati, M. Milani, L. Montorsi, Int. J. Hydrogen Energ. 36, 2803-2816 (2011)

6. M. Milani, L. Montorsi, M. Stefani, Waste Manage. Res. 32, 7, 614-625 (2014)

7. J.M. García-Oliver, X. Margot, M. Chávez, A. Karlsson, Math. Comput. Model. 54, 7-8, 1732-1737 (2011)

8. Y.R.. Lee, H.S. Choi, H.C. Park, J.E. Lee, Comput. Chem. Eng. 82, 202-215 (2015)

9. F. Franzoni, M. Milani, L. Montorsi, SAE Tech. Paper 2008-01-2390 (2008)

10. F.R. Menter, AIAA Paper, No93-2906, 1-21 (1993)

11. R. Siegel, J.R. Howell, Thermal Radiation Heat Transfer (Third Edition, Hemisphere Publishing Co., 1992)

12. J.P. Holman, Heat Transfer (Eighth SI Metric Edition, McGraw Hill, 2001)

13. M. Milani, L. Montorsi, M. Stefani, M. Lizzano, R. Saponelli, J. Environ. Manage. (2017, to be published) 